\title{
Incoercible Vomiting in a Polycystic (ADPKD) Patient on Peritoneal Dialysis
}

\author{
Victor Burguera Vion ${ }^{a}$ b $\quad$ R. Haridian Sosa Barrios ${ }^{a, b}$ \\ Maria Delgado Yagüe ${ }^{a, b}, c \quad$ Milagros Fernández Lucas ${ }^{a, b}$ \\ Maite E. Rivera Gorrína, b, c \\ aHospital Universitario Ramón y Cajal, Madrid, Spain; 'b Instituto Ramón y Cajal de \\ Investigación Sanitaria - IRYCIS, Madrid, Spain; 'Universidad de Alcalá de Henares (UAH), \\ Madrid, Spain
}

\section{Keywords}

Pyloric stenosis - Autosomal dominant polycystic kidney disease - Peritoneal dialysis .

Vomiting

\begin{abstract}
Although gastrointestinal symptoms are not uncommon in PD patients due to several causes, such as infusion volume with early satiety, constipation, or peritonitis, sometimes the differential diagnosis is more challenging for nephrologists. We present the case of a woman with end-stage renal disease due to autosomal dominant polycystic kidney disease on PD who presented with swollen legs and incoercible vomiting. After ruling out constipation and infection, an abdominal CT was done, revealing extrinsic compression of the intrahepatic inferior cava vein (ICV) and massive venous thrombosis from ICV to bilateral iliofemoral deep veins. In addition, CT also showed displacement and extrinsic compression of the stomach, pylorus, and duodenum due to an enlarged liver cyst. Percutaneous drainage and sclerosis of the cyst compressing the stomach was performed, anticoagulation was started, and the patient clinically improved with complete resolution of symptoms.
\end{abstract}




\section{Introduction}

ADPKD is a common cause of renal disease of genetic origin and one of the most frequent causes of ESRD. Last year, 7,142 patients started renal replacement therapy (RRT) in Spain, of which 5.2\% were due to ADPKD [1]. Age and gender are known risk factors for cyst growth [2], and both the liver and kidneys may be differently involved, but surrounding organs are rarely affected by their enlargement. We present the first case of an adult patient on PD due to ADPKD causing pyloric stenosis with severe GI symptoms and limb edema.

\section{Case Report}

A woman in her 40s was diagnosed with ADPKD after a renal ultrasound was performed due to hypertension and deranged kidney function on a routine blood test. At that point, she had a slightly enlarged liver with normal liver function tests and massive renal cysts. She reached ESRD requiring RRT in 2005 and received a deceased donor kidney transplant 2 years later. She developed progressive graft dysfunction due to chronic allograft nephropathy that required starting PD in August 2014. In November 2014, she was admitted to our hospital with a 3-week history of lower limb edema with painful skin ulcers, symptomatic hypotension and tachycardia (118 bpm), anorexia, nausea, and incoercible vomiting. Physical examination revealed a blood pressure of 70/45 $\mathrm{mm} \mathrm{Hg}$ and ulcers with necrotic areas and cellulitis in the external distal third of both legs. Laboratory findings revealed severe malnutrition (serum albumin $1.5 \mathrm{~g} / \mathrm{dL}$ ) and elevated inflammatory markers (C-reactive protein: $288 \mathrm{mg} / \mathrm{L}$; procalcitonin: $1.9 \mu \mathrm{g} / \mathrm{L}$ ). A Doppler ultrasound showed bilateral femoral vein thrombosis, and so pulmonary thromboembolism was ruled out and due to the extensive thrombosis, a complete hypercoagulability state check was done, but no abnormalities were detected and tumor markers were normal. An abdominal CT was then performed showing large liver and renal polycystic disease, both organs with giant cysts $(>15 \mathrm{~cm})$. A giant hepatic cyst producing an extrinsic compression of the intrahepatic inferior cava vein (ICV) and massive venous thrombosis from ICV to bilateral iliofemoral deep veins involving the kidney graft vein was observed. The CT also demonstrated displacement and extrinsic compression of the stomach, pylorus, and duodenum due to another enlarged liver cyst, shown in Figures 1 and 2.

After the CT findings, we considered the clinical presentation was consistent with an ICV syndrome due to ICV compression and thrombosis. Furthermore, vomiting and oral intake intolerance with severe malnutrition were caused by a complete pyloric and duodenal collapse by the enlarged liver cyst.

Fig. 1. Tomography showing extrinsic compression of the intrahepatic inferior cava vein (arrow).

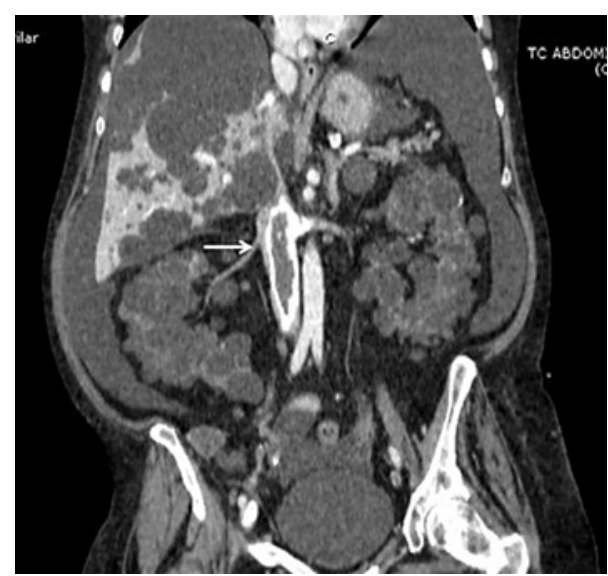


Fig. 2. Tomography showing extrinsic compression of the stomach, pylorus, and duodenum due to an enlarged liver cyst (arrow).

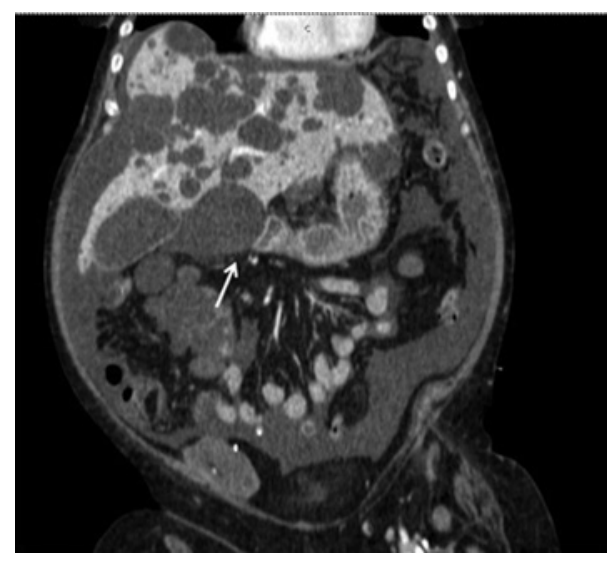

Fig. 3. Tomography showing percutaneous drainage of the compressor liver cyst.

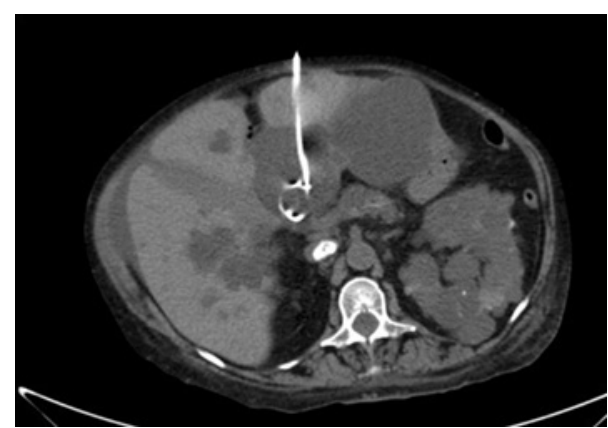

Anticoagulation therapy was started, but vomiting with oral intolerance persisted and the patient remained dependent on parenteral nutrition. Thus, percutaneous drainage and alcohol sclerosis of the compressive liver cyst was decided, with successful return of the stomach to its normal location, shown in Figure 3. Nausea and vomiting resolved in a few days, and adequate oral intake was resumed. Severe hypotension and tachycardia gradually improved, and a repeated CT revealed complete stomach expansion, as shown in Figure 4. PD therapy was modified but not disrupted, and 1 year later, the patient remained asymptomatic. As she did not have any coagulation abnormalities nor any evidence of neoplasia, the massive thrombosis of the ICV was deemed secondary to the extrinsic compression of intrahepatic ICV by enlarged liver cysts.

\section{Discussion}

GI symptoms are frequent in patients with chronic kidney disease, and even more in those receiving renal replacement therapies [3]. Several factors can be accounted for this, including the ESRD causing disease itself. ADPKD is a cilia-related disease of genetic origin and one of the most frequent causes of ESRD in adults.

As a systemic disorder, ADPKD can affect several organs such as the liver, biliary tract, and pancreas, amongst others. In a study by Judge et al. [4], biliary tract and liver complications had a higher incidence in patients undergoing dialysis due to ADPKD (19\% and 15\%, respectively) compared to those on maintenance RRT secondary to other causes, and severe liver complications were more frequent in women, probably related to the fact that there are estrogen receptors in the liver cyst cells. 
Fig. 4. Tomography showing total expansion of the stomach after drainage of the hepatic cyst (arrow).

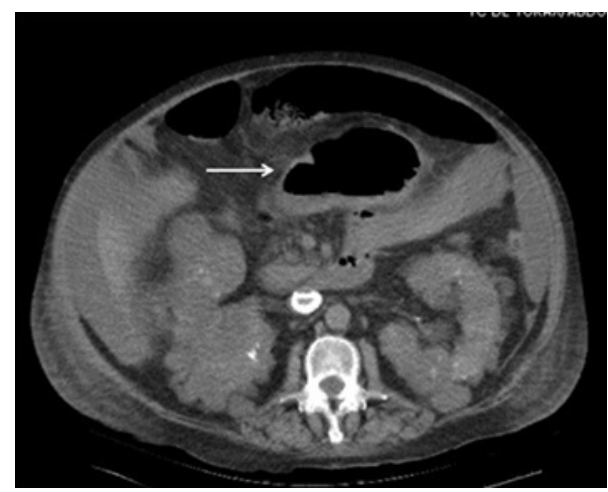

Thrombosis of the intrahepatic ICV secondary to extrinsic venous compression is an uncommon complication described in advanced stages of ADPKD disease [5, 6]. Furthermore, liver cysts may lead to hepatic vein outflow obstruction and Budd-Chiari syndrome with portal hypertension and ascites [6]. Other GI complications observed in advanced stages of ADPKD comprise increased stomach and duodenal pressure with pyrosis due to enlarged kidney size, early satiety, anorexia, and weight loss with nutritional deficiencies $[7,8]$.

In addition, patients on PD therapy may present with diverse GI symptoms, which may complicate a correct diagnosis, such as abdominal discomfort, gastroesophageal reflux, or constipation [9]. Medications, dialysis dosage, dwell volume, and comorbidities contribute to GI alterations; therefore, bearing in mind the original cause of ESRD is key in this complex setting. In some centers, the self-administered questionnaire Gastrointestinal Symptoms Rating Scale (GSRS) [10] has been used to assess their PD patient's perception of GI dysfunction, allowing an early approach to avoid malnourishment and improve the patient's quality of life, as it is a readily available and straightforward tool.

Our patient had ICV thrombosis and pyloric stenosis secondary to her large size hepatic cysts. To our knowledge, pyloric stenosis has not been previously described as a complication of ADPKD, in contrast to children with PKD in whom idiopathic hypertrophic pyloric stenosis has been described [11], and it should be considered in the differential diagnosis of a patient with polycystic kidney disease and persistent vomiting with malnourishment.

With respect to treatment, if symptoms are caused by a large dominant cyst, this may be managed with percutaneous drainage and cyst sclerosis, laparoscopic or open fenestration with variable results. If symptoms are caused by several cysts or liver enlargement, then hepatic resection or orthotopic liver transplantation is the only practical alternative $[12,13]$. In 2 cases reported, long-term anticoagulation alone was an efficient treatment $[14,15]$.

In summary, our case represents a patient with massive liver and kidney ADPKD with giant hepatic cysts that complicated with extensive ICV thrombosis and pyloric stenosis endangering the patient's life. Successful percutaneous treatment completely resolved the symptoms, allowing our patient to restart oral intake, improve her nutritional status, and remain on PD.

\section{Statement of Ethics}

This article does not contain any studies with human participants or animals performed by any of the authors. Ethical approval was not required for this study in accordance with local guidelines. As the patient was deceased and no next of kin was available, informed 
consent to publish this case report was not obtained. All images were anonymized to reduce the likelihood of identification. Extensive attempts to contact the next of kin were unsuccessful. In the absence of refusal of consent to publish this case report by the next of kin, the need for written and informed consent to publish this case report was waived by the Hospital Universitario Ramón y Cajal IRB.

\section{Conflict of Interest Statement}

The authors have no conflicts of interest to declare.

\section{Funding Sources}

No funding was received.

\section{Author Contributions}

V.B.V. contributed to patient care. R.H.S.B. contributed to patient care, writing, and research. M.D.Y.contributed to patient care and draft. M.F.L. contributed to supervision. M.E.R.G. contributed to patient care, conception, supervision, and final approval.

\section{Data Availability Statement}

Data are available upon reasonable request to the corresponding author.

\section{References}

1 Registro de Enfermos Renales de la Sociedad Española de Nefrología. Informe. 2019. Available from: https:// www.senefro.org/modules.php?name=webstructure\&idwebstructure=29 Accessed $2021 \mathrm{Jun} 5$.

2 Cnossen WR, Drenth JP. Polycystic liver disease: an overview of pathogenesis, clinical manifestation and management. Orphanet J Rare Dis. 2014 May 1;9:69.

3 Cano AE, Neil AK, Kang JY, Barnabas A, Eastwood JB, Nelson SR, et al. Gastrointestinal symptoms in patients with end-stage renal disease undergoing treatment by hemodialysis or peritoneal dialysis. Am J Gastroenterol. 2007 Sep;102(9):1990-7.

4 Judge PK, Harper CHS, Storey BC, Haynes R, Wilcock MJ, Staplin N, et al. Biliary tract and liver complications in polycystic kidney disease. J Am Soc Nephrol. 2017;28(9):2738-48.

5 Gabow PA, Johnson AM, Kaehny WD, Manco-Johnson ML, Duley IT, Everson GT. Risk factors for the development of hepatic cysts in autosomal dominant polycystic kidney disease. Hepatology. 1990;11:1033-7.

6 Torres VE, Rastogi S, King BF, Stanson AW, Gross JB Jr, Nogorney DM. Hepatic venous outflow obstruction in autosomal dominant polycystic kidney disease. J Am Soc Nephrol. 1994 Nov;5(5):1186-92.

7 Temmerman F, Missiaen L, Bammens B, Laleman W, Cassiman D, Verslype C, et al. Systematic review: the pathophysiology and management of polycystic liver disease. Aliment Pharmacol Ther. 2011 Oct;34(7):702-13.

8 Fried LF, Palevsky PM, Johnston JR. Duodenal obstruction in polycystic kidney disease. Case report and review of the literature. Am J Nephrol. 1998;18(4):318-20.

9 Dong R, Guo ZY, Ding JR, Zhou YY, Wu H. Gastrointestinal symptoms: a comparison between patients undergoing peritoneal dialysis and hemodialysis. World J Gastroenterol. 2014 Aug 28;20(32):11370-5.

10 Dimenäs E, Glise H, Hallerbäck B, Hernqvist H, Svedlund J, Wiklund I. Quality of life in patients with upper gastrointestinal symptoms. An improved evaluation of treatment regimens? Scand J Gastroenterol. 1993 Aug; 28(8):681-7.

11 Tennakoon J, Koh TH, Alcock G. Pyloric stenosis in a newborn baby with polycystic kidneys. J Perinatol. 2007; 27:125-6. 
Case Reports

in Nephrology

and Dialysis
Case Rep Nephrol Dial 2021;11:321-326 (c) 2021 The Author(s). Published by S. Karger AG, Basel www.karger.com/cnd

12 Macedo FI. Current management of noninfectious hepatic cystic lesions: a review of the literature. World J Hepatol. 2013 Sep 27;5(9):462-9.

13 Russell RT, Pinson CW. Surgical management of polycystic liver disease. World J Gastroenterol. 2007 Oct 14; 13(38):5052-9.

14 Iguchi S, Kasai A, Kishimoto H, Suzuki K, Ito S, Ogawa Y, et al. Thrombosis in inferior vena cava (IVC) due to intra-cyst hemorrhage into a hepatic local cyst with autosomal dominant polycystic kidney disease (ADPKD). Intern Med. 2004 Mar;43(3):209-12.

15 Peces R, Gil F, Costero 0, Pobes A. Massive inferior vena cava thrombosis in a patient with autosomal dominant polycystic hepatorenal disease. Nefrologia. 2002;22(1):75-8. 